\title{
Quantifying Baryon Stopping in High Energy Nuclear Collisions
}

\author{
S.M.H. Wong \\ School of Physics and Astronomy, University of Minnesota, Minneapolis, \\ Minnesota 55455, U.S.A.'
}

\begin{abstract}
We propose a numerical definition for baryon stopping in relativistic heavy ion collisions that is obtainable from final hadron rapidity distributions as well as from bremsstrahlung measurements. Thus a new channel of communication is opened between the two methods.
\end{abstract}

PACS: 25.75.-q, 13.40.-f

Keywords: Heavy Ion Collisions, Baryon Stopping, Bremsstrahlung Emission

1email: swong@nucth1.hep.umn.edu 


\section{Introduction}

Ever since the pioneering scattering experiments by Rutherford in the early 1900s, there has been the persistent question of how matter would behave during very hard collisions. Two examples are the Landau picture where matter would come to a complete stop before exploding due to the enormous pressure built-up or the Bjorken picture where target and projectile would pass through each other experiencing only partial deceleration in the process. Present day high energy nuclear collision experiments provide not only an arena for settling this age-old question but also to see if there is any energy dependence that would change the collision scenario from one picture to another.

Another very good reason to study this is related to the original central goal of these experiments, which is to recreate deconfined matter, the so-called quark-gluon plasma, believed to have existed only in the early universe. To confirm its existence in the laboratory at the up-coming Relativistic Heavy Ion Collider (RHIC) at Brookhaven or the Large Hadron Collider (LHC) at CERN, one relies heavily on finding evidence amongst the many produced particles. These will be affected by the environment in which they are produced. In particular the net baryon number left in the central collision region or the amount of baryon stopping affects, for example, photon and dilepton production. It is therefore of considerable interest to measure this in experiment. Indeed this has been done by NA49 at CERN's SPS [1] by measuring the net proton or baryon rapidity distribution of the final hadrons.

Recently it was proposed [2] to measure this using the bremsstrahlung associated with slowing down of the baryons during the collisions. This method should be simpler than measuring hadron rapidities because of the extreme forward focus of the photons emitted from relativistically moving targets and projectiles. The measurement is therefore much more localized and only photons instead of different types of hadrons need to be detected. From a pragmatic point of view, it is better because most hadron detectors are not able to cover the full rapidity range. From a physical viewpoint, it can say something about the space-time evolution of the collisions [3, 4]. This information will definitely not be available from rapidity measurements alone. In addition, it can distinguish between the Landau and Bjorken picture described above.

In view of the two different ways of measuring baryon stopping, one would like a way to compare the two and be able to communicate between them. In any case, whenever one talks about baryon stopping today, one is usually referring to the shape of the rapidity distribution. As far as we are aware, there is no attempt to quantify it in any way so that the measurement can be put into a more concrete footing. In this paper, we will make

just such an attempt. Whenever we refer to baryon stopping, we are referring to stopping in rapidity space as is traditionally the case and not in velocity space. It will be shown that stopping in rapidity and velocity space are entirely different matters and therefore it is very important that it is made clear in which it is being described. There can be no confusion between the two. 


\section{Quantifying Baryon Stopping or Transparency}

In order to quantify baryon stopping or its inverse, baryon transparency, we aim to find a quantity, called $\mathcal{S}$, that satisfies the following requirements.

(i) It should be equal to unity if there is complete stopping. That is, all baryons end up having $y=0$.

(ii) If there is full transparency, and all final baryons move with the original initial rapidity $y_{0}$, this quantity should be zero.

(iii) One should be able to define its inverse, baryon transparency, $\mathcal{T}$ which is related to $\mathcal{S}$ by the simple relation $\mathcal{S}=1-\mathcal{T}$ so that it has the opposite value in case (i) and (ii).

(iv) $\mathcal{S}$ and $\mathcal{T}$ should both be equal to half or approximately so when it is clear that there is half stopping and half transparency in rapidity space. For example, $\mathcal{S}=\mathcal{T}=1 / 2$ when the rapidity distribution is totally flat.

(v) For different degrees of stopping, $\mathcal{S}$ should range between 0 and 1 , signifying transparent to opaque in that order (or the inverse for $\mathcal{T}$ ).

(vi) It should be obtainable from both hadron rapidity data and from bremsstrahlung measurements. This requirement is essential for bridging the two types of measurements.

A quantity that satisfies all these is

$$
\mathcal{S}=1-\frac{\left(1-v_{0}^{2} \cos ^{2} \theta_{1 / 2}\right)}{2 v_{0}^{2} \cos \theta_{1 / 2}} \int_{-\infty}^{+\infty} d y \frac{v(y) \rho(y)}{1-v(y) \cos \theta_{1 / 2}}
$$

and therefore

$$
\mathcal{T}=\frac{\left(1-v_{0}^{2} \cos ^{2} \theta_{1 / 2}\right)}{2 v_{0}^{2} \cos \theta_{1 / 2}} \int_{-\infty}^{+\infty} d y \frac{v(y) \rho(y)}{1-v(y) \cos \theta_{1 / 2}} .
$$

Here $v_{0}=v\left(y_{0}\right)$ is the initial velocity in the center of mass frame which is related to the initial rapidity $y_{0}$ by the general relation between velocity and rapidity

$$
v(y)=\tanh y
$$

The $\rho(y)$ is proportional to the final baryon rapidity distribution $d N / d y$. For symmetric target and projectile, it is defined by

$$
\int_{-\infty}^{+\infty} d y \rho(y)=2
$$




\begin{tabular}{||l|r|c|c|c||}
\hline Accelerator & $\begin{array}{r}\sqrt{s} / \text { nucleon } \\
{[\mathrm{GeV}]}\end{array}$ & $1-v_{0}$ & $y_{0}$ & $\begin{array}{c}\theta_{1 / 2} \\
(\mathrm{deg})\end{array}$ \\
\hline SPS I & 17.3 & $5.93 \times 10^{-3}$ & 2.91 & 28.83 \\
SPS II & 19.4 & $4.68 \times 10^{-3}$ & 3.03 & 26.97 \\
RHIC & 200.0 & $4.41 \times 10^{-5}$ & 5.36 & 7.93 \\
LHC & 1500.0 & $7.85 \times 10^{-7}$ & 7.38 & 2.87 \\
\hline
\end{tabular}

Table 1: The value of $\theta_{1 / 2}$ at the various accelerators.

To see that specifications (i), (ii) and (iii) are satisfied, one can consider two distributions

$$
\rho(y)=2 \delta(y)
$$

and

$$
\rho(y)=\delta\left(y-y_{0}\right)+\delta\left(y+y_{0}\right)
$$

which correspond to full stopping and full transparency, respectively. It is easily verified that one gets $\mathcal{S}=1, \mathcal{T}=0$ from the first distribution and $\mathcal{S}=0, \mathcal{T}=1$ from the second.

Now for specification (iv), the flat rapidity distribution that respects Eq. (雨) would be

$$
\begin{aligned}
\rho(y) & =1 / y_{0} & & \text { for }|y| \leq y_{0} \\
& =0 & & \text { otherwise . }
\end{aligned}
$$

Using the formula

$$
\int \frac{d y}{1-\cos \theta \tanh y}=\frac{y}{\sin ^{2} \theta}+\frac{\cos \theta}{\sin ^{2} \theta}\{\ln \cosh y+\ln (1-\cos \theta \tanh y)\}
$$

and the requirement that $\mathcal{S}=\mathcal{T}=1 / 2$ for a flat distribution, we end up with the equation

$$
\left(2-v_{0}^{2}\right) \cos \theta_{1 / 2}-v_{0}^{2} \cos ^{3} \theta_{1 / 2}-\frac{\left(1-v_{0}^{2} \cos ^{2} \theta_{1 / 2}\right)}{y_{0}} \ln \left(\frac{1+v_{0} \cos \theta_{1 / 2}}{1-v_{0} \cos \theta_{1 / 2}}\right)=0 .
$$

This equation defines the value of the angle $\theta_{1 / 2}$ which is the angle at which a flat rapidity distribution will yield $\mathcal{S}=\mathcal{T}=1 / 2$ for a given $v_{0}$ or $y_{0}$. At this stage, $\theta_{1 / 2}$ is no more than a numerical quantity but its meaning will be explained below. In Table 1, some values of $\theta_{1 / 2}$ have been computed at the various accelerators. The first case at SPS is for $\mathrm{Pb}+\mathrm{Pb}$ and the second is for $\mathrm{S}+\mathrm{S}$ collisions. The angle becomes smaller as we go to higher energies.

Our specification (iv) requires that rapidity distributions intuitively half way between stopping and transparency in rapidity space should be 0.5. To show that this is indeed 


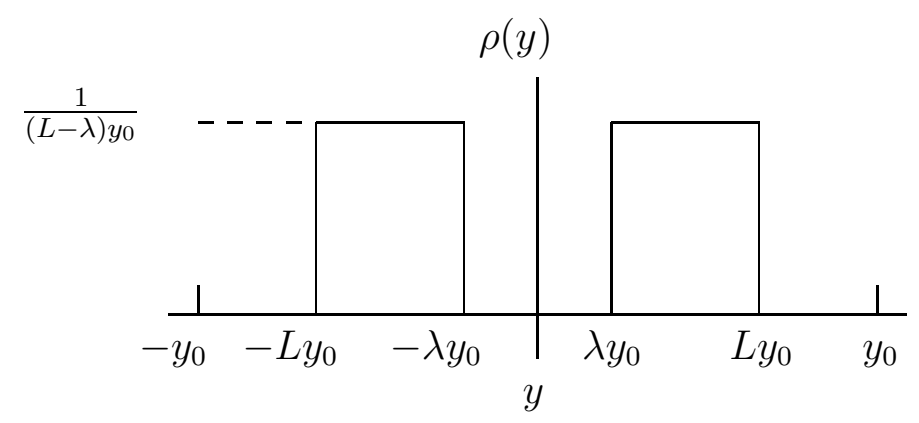

Figure 1: Simple test cases of rapidity distribution used in the text with $1 \geq L \geq \lambda \geq 0$.

the case, we use a simple test distribution depicted in Fig. 1. In this figure, we have two blocks at the height of $1 /(L-\lambda) y_{0}$ because of Eq. (4) and symmetric about $y=0$. The parameters $L$ and $\lambda$ allows us the freedom of a range of distributions. For our purpose here, we set $L=1-\lambda$ and vary the value of $\lambda$. For any value of $\lambda$, we have two blocks symmetric about $\pm y_{0} / 2$, and thus they should all have $\mathcal{S} \sim \mathcal{T} \sim 0.5$. We tabulated the value of $\mathcal{S}$ for a set of values of $\lambda$ at the various accelerators in Table 2 . We recover the flat distribution when $\lambda=0$ and we have two delta functions sitting at $\pm y_{0} / 2$ when $\lambda=1 / 2$. We see that (vi) is better satisfied as we go to higher and higher energies. Since this way of using bremsstrahlung will only be done at RHIC or at LHC, this is good enough and we consider this specification met. In any case, they are all fairly close to 0.5.

One can introduce another special case which is without any doubt half way between opaque and transparent. That is

$$
\rho(y)=\frac{1}{2}\left(\delta\left(y-y_{0}\right)+\delta\left(y+y_{0}\right)+2 \delta\left(y_{0}\right)\right) .
$$

This distribution is artificial but is ideal for our purpose here. This distribution describes half the baryons from the projectile and half from the target sitting at $y=0$ and half of them from each initial nucleus traveling with the original $y_{0}$. This is easily worked out to give the exact result $\mathcal{S}=\mathcal{T}=1 / 2$. The purpose of this last distribution is to show that we have a sensible definition in hand.

\section{Difference Between Rapidity and Velocity Space}

When one looks for a numerical definition for stopping or transparency, one encounters the question of whether this should be in rapidity or velocity space. If one was working within Newtonian mechanics, velocity space would have been the automatic choice. This is quite logical since one could easily associate stopping with the slowing down of the incoming clusters of nucleons. However, it is also traditional to speak of baryon stopping while referring implicitly to the shape of $d N / d y$ in rapidity space. So it is in rapidity space that we gave this definition in the previous section. It must be stressed that the 


\begin{tabular}{||c|c|c|c|c||}
\hline \multirow{2}{*}{$\lambda$} & \multicolumn{4}{|c||}{$\mathcal{S}$} \\
\cline { 2 - 5 } & SPS I & SPS II & RHIC & LHC \\
\hline 0 & 0.50000 & 0.50000 & 0.50000 & 0.50000 \\
$1 / 5$ & 0.49552 & 0.49581 & 0.49921 & 0.49987 \\
$1 / 4$ & 0.49354 & 0.49394 & 0.49881 & 0.49980 \\
$1 / 3$ & 0.49028 & 0.49084 & 0.49801 & 0.49963 \\
$2 / 5$ & 0.48820 & 0.48886 & 0.49836 & 0.49946 \\
$1 / 2$ & 0.48689 & 0.48759 & 0.49689 & 0.49931 \\
\hline
\end{tabular}

Table 2: The value of $\mathcal{S}$ for special test cases of $\rho(y)$ depicted in Fig. 1 with $L=1-\lambda$.
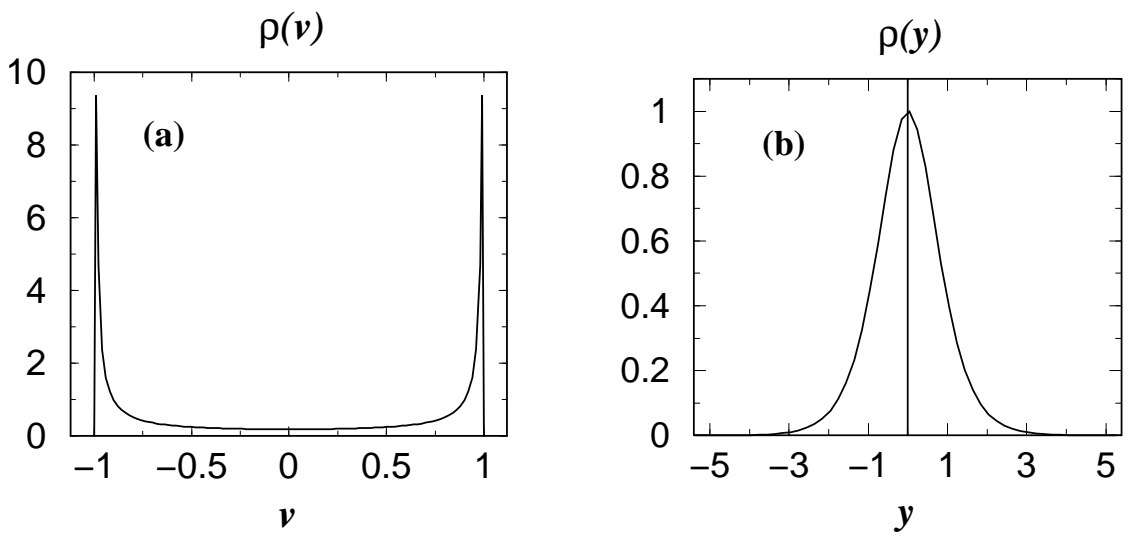

Figure 2: (a) What a flat $\rho(y)$ distribution looks like in velocity space and (b) a flat $\rho(v)$ distribution looks opaque in rapidity space at RHIC.

difference is huge between the two spaces. For example, the flat distribution given in Eq. (7) at RHIC in rapidity space will appear as in Fig. 2 (a) in velocity space because

$$
\rho(y)=\frac{1}{y_{0}} \Longrightarrow \rho(v)=\frac{1}{y_{0}\left(1-v^{2}\right)} \text {. }
$$

The halfway point of stopping in rapidity space at RHIC would appear to be much closer to transparent in velocity space! On the contrary, a flat distribution in velocity space

$$
\rho(v)=\frac{1}{v_{0}} \Longrightarrow \rho(y)=\frac{1}{v_{0} \cosh ^{2} y}
$$

would be more opaque than transparent when one switches to rapidity. This is shown in Fig. 2 (b). Using this distribution, one finds $\mathcal{S} \simeq 0.97$ or $\mathcal{T} \simeq 0.03$. For this reason, we have insisted that our definition be in rapidity space, conforming to the convention used in the heavy ion collision community. 


\section{How To Obtain $\mathcal{S}$ and $\mathcal{T}$ From Bremsstrahlung Measurements}

In Sect. 2, the definition given for baryon stopping $\mathcal{S}$ and transparency $\mathcal{T}$ depended on the distribution $\rho(y)$ which, for symmetric collisions, is related to the net baryon rapidity distribution by $d N_{B-\bar{B}} / d y=A \rho(y)$ or net proton rapidity distribution by $d N_{p-\bar{p}} / d y \simeq$ $Z \rho(y)$. Here $Z$ and $A$ are the atomic and mass number respectively of the incoming target or projectile. This assumes that the net proton rapidity distribution is representative of or approximately proportional to the net baryon distribution. This does not seem to be too bad an assumption if one examines data from SPS [1]. Since one could easily calculate the value of $\mathcal{S}$ and $\mathcal{T}$ for some given data of $d N / d y$, the important question would be how to meet specification (vi) in Sect. 2. The main difficulty is how to obtain the quantity $\mathcal{S}$ or $\mathcal{T}$ from bremsstrahlung measurements. To solve this problem, we now state that $\mathcal{S}$ can be related to the intensity distribution of the bremsstrahlung emitted in nuclear collisions by

$$
\mathcal{S}=\frac{1-v_{0}^{2} \cos ^{2} \theta_{1 / 2}}{v_{0}^{2} \sin 2 \theta_{1 / 2}}\left(\left.\frac{4 \pi^{2}}{\alpha Z^{2}} \frac{d^{2} I}{d \omega d \Omega}\right|_{\substack{\omega \rightarrow 0 \\ \theta=\theta_{1 / 2}}}\right)^{1 / 2} .
$$

The numerical quantity $\theta_{1 / 2}$ has now been given the physical meaning of the opening angle from the beam pipe in which direction the soft photons should be measured. To see exactly where this angle lies in relation to other directions, one can work out the angle of maximum intensity assuming, for example, the case of full stopping $\mathcal{S}=1$ or complete opacity $\mathcal{T}=0$ using the formulae given in ref. [1]. In this case, the cosine of this angle $\theta_{\max }$ is related to the initial velocity $v_{0}$ by

$$
\cos \theta_{\max }=\left(2-v_{0}^{2}\right)^{-1 / 2}
$$

It then works out at RHIC to be $\theta_{\max }=0.538^{\circ}$ and $\theta_{\max }=0.072^{\circ}$ at $\mathrm{LHC}$. In view of the fact that the intensity distribution falls off with the opening angle $\theta$ from the beam pipe, these $\theta_{\max }$ are not too far from those $\theta_{1 / 2}$ in Table 1 at the respective accelerators so that there will be sufficient intensity at the $\theta_{1 / 2}$ to enable the photon measurements.

If the reader has not guessed it already, we will now disclose the physical meaning of the somewhat mysterious quantity $\mathcal{S}$ or $\mathcal{T}$. The inverse of the prefactor to the rapidity integral in Eq. (2) is in fact proportional to the radiation amplitude for full stopping [4]. So one can now see why the rapidity integral itself will always be less than or equal to the inverse of this prefactor (but see the next paragraph concerning the soft photon requirement). Because of $\mathcal{S}$ and $\mathcal{T}$ have an origin in the bremsstrahlung intensity distribution, they depend on the (charge) hadron rapidity distribution which automatically allows them to bridge the two different methods of determining baryon stopping. So far we have not mentioned the contribution from charged mesons to photon emissions. They could potentially ruin Eq. (13). However, pions are by far the most abundant meson 


\begin{tabular}{||cccc|c|cccc||}
\hline$L$ & $\lambda$ & $\mathcal{S}$ & $\mathcal{T}$ & & $\lambda$ & $\lambda$ & $\mathcal{S}$ & $\mathcal{T}$ \\
\hline $1 / 5$ & 0 & 0.991 & 0.009 & & $1 / 2$ & $1 / 3$ & 0.700 & 0.300 \\
$1 / 3$ & 0 & 0.965 & 0.035 & & $3 / 4$ & $1 / 3$ & 0.417 & 0.583 \\
$1 / 2$ & 0 & 0.876 & 0.123 & & $4 / 5$ & $1 / 3$ & 0.377 & 0.622 \\
\cline { 5 - 7 } $3 / 4$ & 0 & 0.661 & 0.339 & & $3 / 4$ & $1 / 2$ & 0.229 & 0.771 \\
$4 / 5$ & 0 & 0.622 & 0.377 & & $4 / 5$ & $1 / 2$ & 0.198 & 0.801 \\
\cline { 1 - 4 } $1 / 3$ & $1 / 5$ & 0.926 & 0.074 & & 1 & $1 / 2$ & 0.124 & 0.876 \\
\cline { 5 - 7 } $1 / 2$ & $1 / 5$ & 0.800 & 0.200 & & $4 / 5$ & $3 / 4$ & 0.045 & 0.954 \\
$3 / 4$ & $1 / 5$ & 0.540 & 0.460 & & 1 & $3 / 4$ & 0.018 & 0.982 \\
\cline { 5 - 7 } $4 / 5$ & $1 / 5$ & 0.499 & 0.501 & & 1 & $4 / 5$ & 0.011 & 0.989 \\
\hline
\end{tabular}

Table 3: The values of $\mathcal{S}$ and $\mathcal{T}$ for some more general test cases of $\rho(y)$ depicted in Fig. 1 with various $L$ and $\lambda$ at RHIC energies.

type and they can be positively as well as negatively charged. Therefore contribution to bremsstrahlung from mesons cancel out to a large extent [5].

Although it has been expressed in Eq. (13) that the intensity distribution should be for low energy photons, in practice a few to tens of $\mathrm{MeV}$ should be good enough. The reason for the soft photon requirement is to remove all nuclear structural dependence as well as any potential interference effects. In ref. [4] it was shown that if there were more than one component in the acceleration of the nuclear clusters during the collisions, this would result in enhancement in and oscillations of the intensity distribution $d I / d \omega d \Omega$ with $\omega$. This is a direct result of the interference between the various components in the acceleration. For the purpose of our definition, interference would unfortunately just taint any value of $\mathcal{S}$ obtained from Eq. (13). Only in the soft $\omega$ limit is it free from this type of effect. As seen in Fig. 7 of ref. [4], the intensity at small $\omega$ is invariant under this.

\section{$5 \quad$ Examples}

We will now try some example distributions and work out their $\mathcal{S}$ and $\mathcal{T}$ values. For the test distribution in Fig. 1 we vary the two parameters $L$ and $\lambda$ to obtain the stopping values for the different cases. The first five entries in Table 3 are for central single-block distributions centering around $y=0$. As the distribution is widened, $\mathcal{S}$ decreases towards the flat 0.5 value, as expected. The subsequent groups of entries are for symmetric twoblock distributions shifting progressively away from the center to either side towards $|y|=1$. Thus in each group there is the tendency $\mathcal{S} \rightarrow 0$ and $\mathcal{T} \rightarrow 1$ which is how a sensible definition should behave.

Admittedly these distributions are only test cases designed to show how the numerical definition works. However, more realistic distributions can always be approximately 
reconstructed from thin blocks (strips) of varying heights so the simple distributions used do not affect in any way how the definition meets the specifications stipulated in Sec. 2 . It may be that in practice our definition would need to be refined but here we have laid the groundwork for a simple but sensible numerical definition for baryon stopping. For the actual applications of this to real data, and for other ways of using bremsstrahlung from more realistic collision models than those used in [4], we refer the reader to [5].

\section{Acknowledgments}

The author would like to thank Joe Kapusta for a critical reading of the manuscript and for discussions. This work was supported by the U.S. Department of Energy under grant DE-FG02-87ER40328.

\section{References}

[1] NA49 collaboration, Phys. Rev. Lett. 82 (1999) 2471.

[2] S. Jeon, J. Kapusta, A. Chikanian and J. Sandweiss, Phys. Rev. C 58 (1998) 1666.

[3] J.I. Kapusta and S.M.H. Wong, Proceedings of the XXIX International Symposium on Multiparticle Dynamics, August 1999, preprint NUC-MINN-99/15-T, hepph/9909573.

[4] J.I. Kapusta and S.M.H. Wong, Phys. Rev. C 59 (1999) 3317.

[5] Work in progress. 